\title{
Isolation of mimotopes to the anti-human VEGF antibody Bevacizumab by mRNA display using random peptide libraries and the vaccination of a rabbit
}

\author{
Teruaki Kobayashi, Tatsuro Shibui*
}

Molecuence Corporation, Mitsubishi Chemical Group Yokohama Research Center, Yokohama, Japan.

Email: shibui_at_home@yahoo.co.jp

Received 8 February 2011; revised 20 March 2011; accepted 1 April 2011.

\begin{abstract}
An mRNA display system using synthetic DNA coding for random 10- and 20-amino-acid peptide libraries was employed to isolate mimotopes that could substitute for the anti-human VEGF antibody Bevacizumab. After six rounds of affinity selection, three clones that bound to the antibody were isolated. Their random-peptide portions were chemically synthesized, and further characterized. All of the peptides showed clear specific binding to the antibody. Two of them were further conjugated with Keyhole limpet hemocyanin (KLH) to immunize a rabbit. After five immunizations biweekly, antibodies to the peptides were purified with a column conjugated with the peptides. The purified antibodies reacted specifically to the antibody's original antigen, human VEGF. mRNA displays could be useful for the isolation of mimotopes for vaccines to substitute for therapeutic antibodies.
\end{abstract}

Keywords: Random Peptide; mRNA Display; Mimotope; Antigen; Antibody; Vaccine

\section{INTRODUCTION}

In vitro peptide or protein display systems [1,2] have developed with advances in cell-free translation systems such as E. coli cell lysate [3,4] and wheat embryo extract $[5,6]$. Compared with in vivo systems such as phage display [7] and yeast surface [8] display, the in vitro systems can utilize larger libraries since they do not require the transformation of living cells. Theoretically, $1 \times 10^{13}$ independent clones can be used for screening. Ribosome and mRNA displays are commonly employed as in vitro protein display systems $[1,9,10]$. Ribosome displays are simpler but less stable than mRNA displays. The peptide portion in ribosome displays is attached to the mRNA through a ribosome, whereas in mRNA displays, in vitro-synthesized peptides are chemically conjugated to the mRNAs via puromycin, a peptidyl acceptor antibiotic [1,9,11]. A covalent bond can be generated between the peptide and mRNA through translation in vitro with synthetic mRNA that carrying puromycin at the 3' end. During selection, the covalent bond stabilizes the peptide-mRNA complex, although the process of making the display molecules is more complicated.

Bevacizumab [12], a humanized monoclonal antibody produced by recombinant Chinese Ovary Cells [13], recognizes and blocks vascular endothelial growth factor A (VEGF-A). VEGF-A is a protein that triggers a chemical signal stimulating the growth of new blood vessels (angiogenesis), especially in cases of cancer, retinal proliferation of diabetes in the eye, and other diseases. Bevacizumab is used to treat cancers that are metastatic. However, as it is produced by mammalian cells as a recombinant protein, it is very expensive. Cost-effective substitutes for the antibody are needed.

In many monoclonal antibodies, phage display systems with random peptide libraries (RPL) have been used for epitope mapping [7]. In cases of antibodies that recognize conformational epitopes, the systems have often provided binding peptides with no homology to the amino acid sequences of the original antigens [14]. Such peptides are called mimotopes [15]. Several mimotopes have been shown to induce the production of antibodies against the original antigen $[15,16]$. These new antibodies seemed to recognize a similar or the same epitope as the original antibody [16]. Thus mimotopes could potentially be used as substitutes for expensive therapeutic antibodies [17]. Compared to phage displays, mRNA displays provide a larger library, and would increase the chance of identifying mimotopes.

In this paper, we describe the successful application 
of an mRNA-display system with random linear 10-and 20-amino-acid peptide libraries to the isolation of mimotope peptides to a therapeutic antibody, Bavacizumab, and their possible use for a vaccine.

\section{MATERIALS AND METHODS}

\subsection{Antibodies}

Abciximab (ReoPro), Cetuximab (Erbitux), Adalimumab (Humira) and Omalizumab (Xolair) were purchased from Eli Lilly (Switzerland), Merck (Switzerland), Abbott (Switzerland) and Novartis (Switzerland), respectively. Tocilizumab (Actermura) and Bevacizumab (Avastin) were obtained from Roche (Switzerland). Human IgGs was obtained from Sigma (USA).

\subsection{Construction of mRNA Display Libraries}

DNA fragments for the linear random 10- and 20- amino acid libraries, RL10aPL and RL20aPL, were synthesized by BEX (Tokyo, Japan). The mRNA display libraries were constructed separately using the DNA templates as described previously [14]. The sequences of the peptide portion of mRNA display molecules are as follows.

RL10aPL;

MGXXXXXXXXXXGGSGG-

DYKDDDDKGGSSSGRGAAG,

RL20aPL;

MGXXXXXXXXXXXXXXXXXXXXGGSGG-

DYKDDDDKGGSSSGRGAAG,

in which $\mathrm{X}$ is a random amino acid.

\subsection{Affinity Selection}

To prevent non-specific bindings to the antibody, the mRNA display molecules (in $200 \mu \mathrm{l}$ of a washing buffer (50 mM Tris/HCl $\mathrm{pH} 7.5,150 \mathrm{mM} \mathrm{NaCl}$, and $0.1 \%$ Tween 20) containing a final concentration of $0.05 \%$ bovine serum albumin and $25 \mu \mathrm{g} / \mathrm{ml}$ tRNA) were incubated with $10 \mu \mathrm{l}$ of human normal IgG (Sigma)-bound Protein G-Sepharose beads (GE Healthcare) for 30 minutes at room temperature with rotation. The supernatant, the non-bound fraction, was transferred to $10 \mu$ l of the Bevacizumab -bound Protein G-Sepharose beads and rotated for 1 hour at room temperature. The beads were washed three times with $0.1 \mathrm{ml}$ of the washing buffer (50 mM Tris/HCl $\mathrm{pH}$ 7.5, $150 \mathrm{mM} \mathrm{NaCl}$, and 0.1\% Tween 20), and the bound molecules were eluted two times with $40 \mu \mathrm{l}$ of $0.1 \mathrm{M}$ Glycin- $\mathrm{HCl} \mathrm{pH} 2.5$, and immediately neutralized with $8 \mu \mathrm{l}$ of $1 \mathrm{M}$ Tris- $\mathrm{HCl} \mathrm{pH} 8.0$. The eluted molecules were re-amplified by PCR for the next round of selection. Subsequent rounds were performed in a similar manner.

\subsection{Cloning and Sequencing}

After six selection cycles, PCR-amplified fragments coding for bound peptides were cloned into a vector (pGEM T Easy Vector, Promega), and sequenced. The sequence of each clone was sorted using the CLUSTALW Program.

\subsection{Synthetic Peptides}

Random peptide-portions from isolated clones were chemically synthesized, and purified with HPLC by Toray Research Center (Kanagawa, Japan)

\subsection{Immunization of Peptides}

The peptides of two isolated clones, M074_F02 and M074_D12 (see Table 1), were conjugated with keyhole limpet hemocyanin (KLH), and injected into a rabbit in every two weeks (five times in total) by Scrum (Tokyo, Japan).

\subsection{Purification of Antibodies against Peptides}

C-terminally biotinylated M074_F02 and M074_D12 were synthesized by Scrum. First, $50 \mu$ l of NeutrAvidin Agarose (Thermo Scientific) was placed in a spin column and washed three times with $200 \mu$ of PBST $(1 \times$ PBS and $0.05 \%$ Tween 20). Then, $100 \mu$ l of $10 \mu \mathrm{M}$ biotinylated M074_F02 and M074_D12 in PBS was added and the column was incubated at $4{ }^{\circ} \mathrm{C}$ overnight to make peptide-immobilized beads. The beads were washed three times with $200 \mu \mathrm{l}$ of PBST. Next, $100 \mu \mathrm{l}$ of serum from the immunized rabbit was diluted in $400 \mu \mathrm{l}$ of PBST. The diluted serum was mixed with the M074_F02 and M074_D12-immobilized beads for 30 minutes at room temperature. The beads were washed three times with PBST. The bound antibodies were eluted with 100 $\mu \mathrm{l}$ of $10 \mathrm{mM}$ Glycin-HCl (pH 2.7), and immediately neutralized by adding $11 \mu \mathrm{l}$ of $1 \mathrm{M}$ Tris-HCl ( $\mathrm{pH}$ 8.9). These fractions were used to monitor the antibodies against the original antigen, human VEGF.

Total serum was collected one week after the final immunization, and its IgG fraction was purified with a Protein A column by Scrum. Then the aliquots of approximately $1 \mathrm{ml}$ were further purified separately with the M074_F02 and M074_D12-immobilized beads as described above. The bound fraction of each peptide was used to analyze reactivity to the original antigen by ELISA.

Table 1. A list of clones isolated.

\begin{tabular}{ccc}
\hline Clone \# & Amino acid sequence & $\begin{array}{c}\text { Appearance } \\
\text { rate (\%) }\end{array}$ \\
\hline M074_F02 & KLEMHFPSHVISVADGWSLF & 5 \\
M074_D12 & WLEMHWPAHS & 68 \\
M075_A10 & RDLRHCESSWHKLVDFYCYT & 27 \\
\hline
\end{tabular}




\section{RESULTS AND DISCUSSION}

\subsection{Amino Acid Sequence of Bound Molecules}

Amino acid sequences of the random peptide portion of isolated molecules are listed in Table 1. Of the 92 clones sequenced, the most dominant was M074_D12. Although M075_A10 was isolated from the 20-aa linear peptide library, it contains two cysteine residues. These clones did not show any apparent homology in sequence.

\subsection{Binding to Bevacizumab}

To clarify whether the polypeptide portions of the mRNA-display molecules bound to the antibody, the polypeptides were translated in vitro and labeled at the C-terminus with Cy3 instead of mRNA using puromycin. The binding of the labeled polypeptides to Bevacizumab was then examined by pull-down assay as described in the legend to Figure 1. All of the clones showed specific binding to the antibody. Unexpectedly, the most dominant clone, M074_D12, did not show the highest intensity or specificity of binding.

\subsection{Binding Properties of the Peptides}

Peptides from random portions in isolated clones were

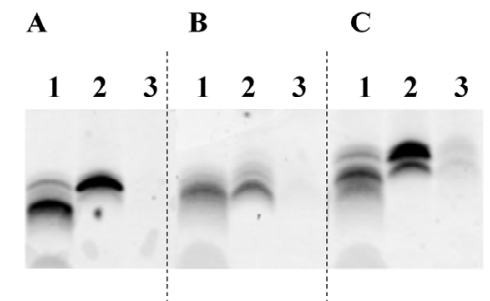

Figure 1. Pull-down assay of isolated clones with Bevacizumab. A Clone M074_F02, B Clone M074_D12, and C Clone M075_A10. C-terminally Cy3-labeled polypeptides were mixed with Bevacizumab-bound Protein G beads and normal IgG-bound beads as a negative control. The beads were spun down by brief centrifugation and washed three times with binding buffer. The bound polypeptides were eluted with $0.1 \mathrm{M}$ Glycin- $\mathrm{HCl}(\mathrm{pH} 2.5)$ and neutralized by $1 \mathrm{M}$ Tris-buffer $(\mathrm{pH}$ 8.0) (See details in Materials and Methods). Labeled polypeptides were separated on a Tricin polyacrylamide gel by electrophoresis and detected through Cy3 fluorescence. Lane 1: ten percent of the labeled polypeptide was loaded on the gel. Lane 2: the labeled polypeptide eluted from the Bevacizumab-bound beads. Lane 3: negative control. The labeled polypeptide eluted from the normal human IgG-bound beads. synthesized chemically and purified with HPLC. Figure 2 shows the inhibition of antigen binding to the antibody. All of them showed similar patterns. The results did not indicate higher affinity of M074_D12. The specificity of the peptides was examined further using other therapeutic antibodies (Figure 3). All of them bound specifically to Bevacizumab. M074_D12 showed slightly higher binding than the others.

\subsection{Influence of ReducingConditions on Binding}

Next, we examined whether the two cysteine residues in M075_A10 formed a di-sulfide bond and were essential for binding. Under reducing conditions, M075_A10 lost binding activity (Figure 4). It seemed to form a circular structure, however, this remains to be confirmed. To avoid this structural complication, we chose M074_F02 and M074_D12 for immunization.

\subsection{Immunization of the Rabbit and Characteristics of its Serum}

To minimize number of animals to be sacrificed, M074_F02-KLH and M074_D12-KLH conjugates were mixed together in adjuvants, and injected into a rabbit five times at two-week intervals.

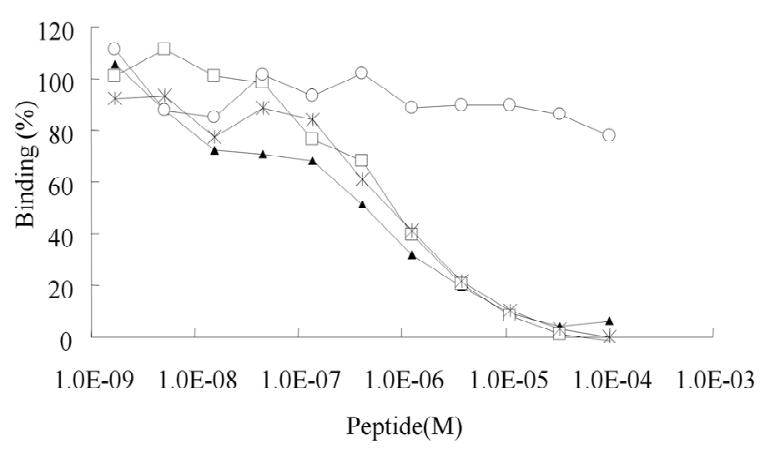

Figure 2. Inhibition of antigen-antibody binding by synthetic peptides of isolated clones. $\mathbf{\Delta}$ : M074_F02, $\square$ : F074_D12, * : M075_A10, and $\bigcirc$ : control. The random peptide portion of each clone was chemically synthesized by Toray Research Center (Japan). Assays were conducted essentially as described previously [10]. Aliquots $(100 \mu \mathrm{l})$ of Bevacizumab solution ( $3 \mu \mathrm{g} / \mathrm{ml}$ in PBS) were added to wells of ELISA plates (COSTAR 9018), and incubated at $4^{\circ} \mathrm{C}$ overnight. The plates were washed 3 times with PBS. The wells were blocked with $1 \%$ BSA in PBS at $37^{\circ} \mathrm{C}$ for 2 hours. The plates were washed three times with PBST. Peptides of the indicated concentration $(100 \mu \mathrm{l})$ were incubated with human VEGF solution (70 ng/ml in PBS, PeproTech) in the Bevacizumab-coated wells at room temperature for 1 hour. Bound human VEGF was detected with a rabbit anti-human VEGF, and then bound anti-human VEGF was detected with anti-rabbit IgG conjugated with HRP (Sigma). Inhibition of binging (\%) was calculated from absorbance at $490 \mathrm{~nm}$ using 1.2-Phenylenediamine in Citrate buffer containing $\mathrm{H}_{2} \mathrm{O}_{2}$. 


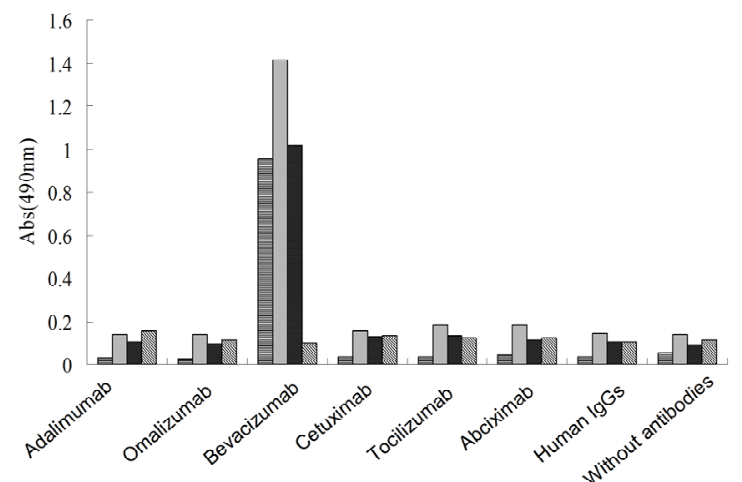

Figure 3. Specificity of the peptides; $\square$ M074_F02; M074_D12; M075_A10; Negative control. Binding to therapeutic antibodies was assayed by ELISA. All antibodies were purchased (see Materials and Methods). Aliquots (100 $\mu \mathrm{l})$ of each antibody solution ( $3 \mu \mathrm{g} / \mathrm{ml}$ in PBS) were added to wells of ELISA plates (COSTAR 9018), and incubated at $4^{\circ} \mathrm{C}$ overnight. The plates were washed three times with PBS. The wells were blocked with $1 \%$ BSA in PBS at $37^{\circ} \mathrm{C}$ for 2 hours. The plates were washed 3 times with PBST. Aliquots $(100 \mu \mathrm{l})$ of C-terminally biotin-labeled peptides $(10 \mu \mathrm{M}$ in PBS) were put into the wells, and incubated for 1 hour at room temperature. The plates were again washed three times with PBST. Next, $100 \mu \mathrm{l}$ of 200-fold diluted streptavidin conjugated with HRP (R\&D systems) was added to the wells and incubation was continued for 1 hour at room temperature. The plates were again washed three times with PBST. Bound peptides were detected by measuring absorbance at $490 \mathrm{~nm}$ using 1.2-Phenylenediamine in Citrate buffer containing $\mathrm{H}_{2} \mathrm{O}_{2}$.
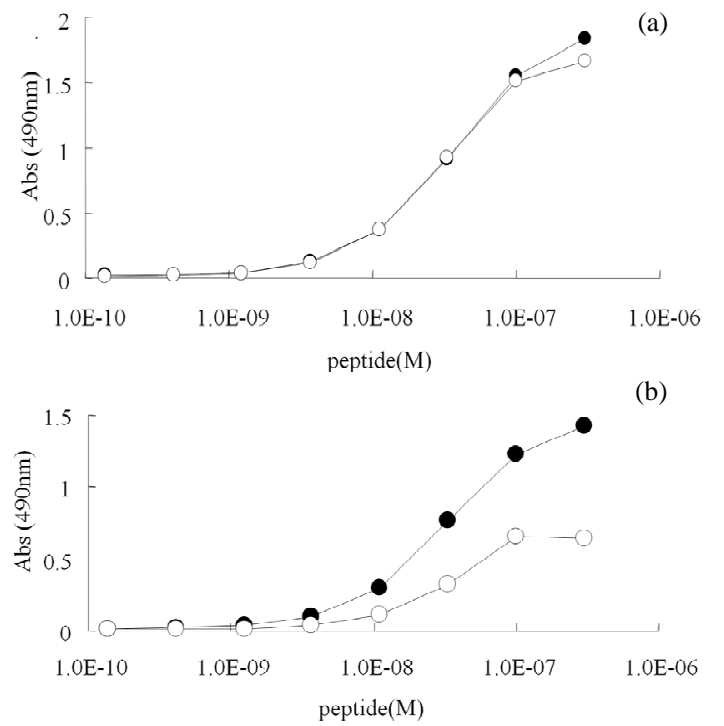

Figure 4. Effect of a reducing condition on binding to Bevacizumab. (a) M074_D12. (b) M075_A10. M074_D12 and M075_A10 were incubated with (-○-) or without (--) 100 mM dithiothreitol (DTT) in PBS for 3 hours at room temperature prior the assay. Indicated amounts of peptides were incubated with Bevacizumab-coated plates (See Figure 2 legend). Assays were performed essentially as described in Figure 3.

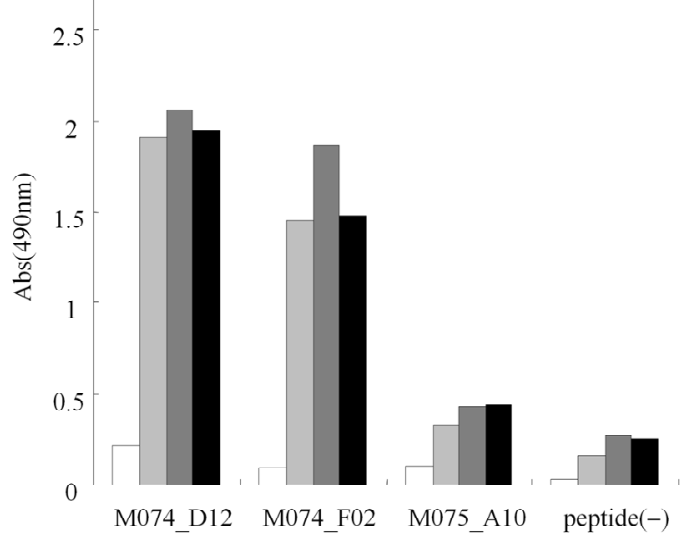

Figure 5. Serum titers against each peptide. Serum titers against each peptide in a rabbit immunized with peptide-KLH conjugates were assayed with ELISA. Sera were collected prior to immunization $(\square)$ and one week after the 3rd ( $\square$ ), 4th ( $\square$ ) and final $(\square)$ immunization. Aliquots $(100 \mu \mathrm{l})$ of peptide solution (1 $\mu \mathrm{M}$ in PBS) were added to wells of a NeutrAvidin coated plate (Thermo Scientific), and incubated at $4^{\circ} \mathrm{C}$ overnight. The plate was washed 3 times with PBS. The wells were blocked with $1 \%$ BSA in PBS at $37^{\circ} \mathrm{C}$ for 2 hours. The plate was washed 3 times with PBST. $100 \mu \mathrm{l}$ of rabbit serums diluted $10^{3}$-fold in PBS was put into each well, and incubation was continued for 1 hour at room temperature. The plate was again washed 3 times with PBST. Next, $100 \mu \mathrm{l}$ of $10^{4}$-fold diluted anti-rabbit IgG conjugated with HRP (Sigma) was added to each well and incubated for 1 hour at room temperature. The plate was washed 3 more times with PBST. Bound rabbit IgGs were detected by measuring absorbance at $490 \mathrm{~nm}$ using 1.2-Phenylenediamine in Citrate buffer containing $\mathrm{H}_{2} \mathrm{O}_{2}$.

Serum titers were monitored one week after the 3rd, 4th and 5th (final) immunizations (Figure 5). Titers against immunized peptides were specifically raised almost the same manner.

The serum samples from the rabbit one week after the 3rd and 4th immunizations were further affinity-purified on a column packed with both M074_F02 and M074_ D12-conjugated beads to monitor their reactivity to human VEGF and other antigens (Figure 6). Specific antibodies against human VEGF were successfully raised in the rabbit.

Blood was collected one week after the final immunization. Titers against each peptide in the final serum sample are shown in Figure 5. The final serum sample was firstly purified with a Protein A column, and further affinity purified on columns separately packed with beads conjugated with M074_F02 and M074_ D12. The 


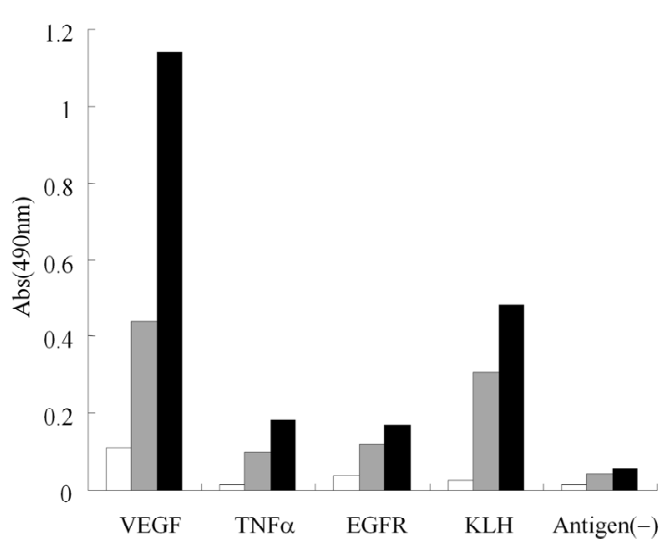

Figure 6. Monitoring of the binding of antibodies to human VEGF during immunization; $\square$ Pre-immune serum; $\square$ One week after the 3rd immunization; $\square$ One week after the 4th immunization. Serum from a rabbit immunized with the peptide-KLH conjugates was affinity-purified with a peptide (M074_D12 and M075_ A10)-conjugated column as described in Materials and Methods. Purified fractions were diluted 10-fold, and assayed for reactivity to human VEGF with ELISA as described in Figure 5 except the plate was coated with the proteins indicated instead of peptides
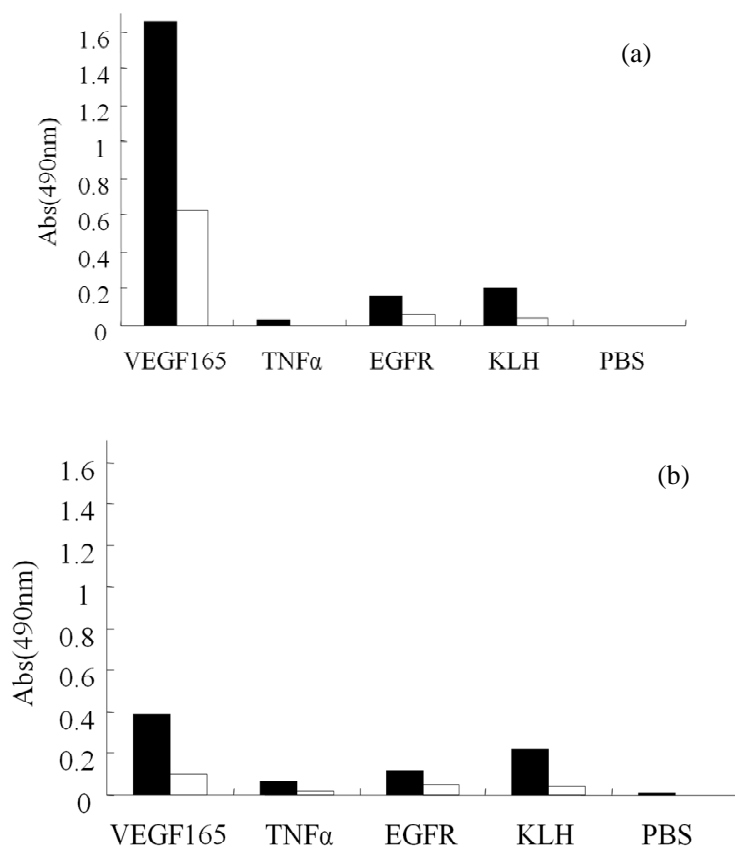

Figure 7. Binding activity of antibodies purified separately with M074_D12 and M075_A10-conjugated columns. (a) Antibodies purified with the M074_D12-conjugated column. (b) Antibodies purified with the M075_ A10-conjugated column; 10-fold dilution of the purified fraction; 50-fold dilution of the purified fraction. Assays were done essentially as described in Figure 6. purified fractions were examined for reactivity to human VEGF and other antigens (Figure 7). They both bound specifically to human VEGF. The antibodies purified with M074_ D12 showed more reactivity to the antigen than those purified with M074_F02 (Figure 7). M074_D12 seems to be more similar to Bevacizumab's epitope than the other mimotope.

\section{CONCLUSIONS}

Three mimotopes against Bvacizumab were isolated display system with random peptide libraries. Chemically synthesized peptides of the mimotopes reacted similarly to the antibody in ELISAs. One of them (M074_D12) generated antibodies to Bevacizumab's original antigen, human VEGF, in a rabbit. The results indicate that mRNA displays can be used to isolate peptide mimotopes to substitute for therapeutic antibodies.

\section{REFERENCES}

[1] Lipovsek, D. and Pluckthun, A. (2004) In-vitro protein evolution by ribosome display and mRNA display. Journal of Immunological Methods, 290, 51-67. doi:10.1016/j.jim.2004.04.008

[2] Shibui, T., Kobayashi, T. and Shiratori, M. (2008) Isolation of cross-reacting antigen candidates by mRNAdisplay using a mixed cDNA library. Biotechnology Letters, 30, 2037-2043. doi:10.1007/s10529-008-9803-5

[3] Shimizu, Y., et al. (2001) Cell-free translation reconstituted with purified components. Nature Biotechnology, 19, 751-755. doi:10.1038/90802

[4] Kim, D.M. et al. (1996) A highly efficient cell-free protein synthesis system from Escherichia coli. European Journal of Biochemistry, 239, 881-886. doi:10.1111/j.1432-1033.1996.0881u.x

[5] Koga, H., Misawa, S. and Shibui, T. (2009) A wheat embryo cell-free protein synthesis system not requiring an exogenous supply of GTP. Biotechnology Progress, 25, 1322-1327. doi:10.1002/btpr.230

[6] Madin, K., et al. (2000) A highly efficient and robust cell-free protein synthesis system prepared from wheat embryos: plants apparently contain a suicide system directed at ribosomes. Proceedings of the National Academy of Sciences of the United States of America, 97, 559-564. doi:10.1073/pnas.97.2.559

[7] Smith, G.P. and Scott, J.K. (1993) Libraries of peptides and proteins displayed on filamentous phage. Methods in Enzymology, 217, 228-257. doi:10.1016/0076-6879(93)17065-D

[8] Furukawa, H., et al. (2006) Development of novel yeast cell surface display system for homo-oligomeric protein by coexpression of native and anchored subunits. Biotechnology Progress, 22, 994-997. doi:10.1021/bp0601342

[9] Nemoto, N., et al. (1997) In vitro virus: Bonding of mRNA bearing puromycin at the 3'-terminal end to the C-terminal end of its encoded protein on the ribosome in 
vitro. FEBS Letters, 414, 405-408. doi:10.1016/S0014-5793(97)01026-0

[10] Shibui, T., et al. (2009) In vitro selection of scFv and its production: An application of mRNA display and wheat embryo cell-free and E. coli cell production system. Applied Microbiology and Biotechnology, 84, 725-732. doi:10.1007/s00253-009-2010-z

[11] Miyamoto-Sato, E., et al. (2000) Specific bonding of puromycin to full-length protein at the C-terminus. Nucleic Acids Research, 28, 1176-1182. doi:10.1093/nar/28.5.1176

[12] Chen, H.X., Gore-Langton, R.E. and Cheson, B.D. (2001) Clinical trials referral resource: Current clinical trials of the anti-VEGF monoclonal antibody bevacizumab. Oncology (Williston Park), 15, 1023-1026.

[13] Kito, M. et al. (2002) Construction of engineered CHO strains for high-level production of recombinant proteins. Applied Microbiology and Biotechnology, 60, 442-448. doi:10.1007/s00253-002-1134-1
[14] Shiratori, M., Kobayashi, T. and Shibui, T. (2009) Identification of amino acids essential for antibody binding by mRNA-display using a random peptide library: an anti-human tumor protein p53 antibody as a model. Molecular Biotechnology, 41, 99-105. doi:10.1007/s12033-008-9113-0

[15] Riemer, A.B., et al. (2004) Generation of Peptide mimics of the epitope recognized by trastuzumab on the oncogenic protein Her-2/neu. Journal of Immunology, 173, 394-401.

[16] Saphire, E.O., et al. (2007) Structure of a high-affinity "mimotope" peptide bound to HIV-1-neutralizing antibody b12 explains its inability to elicit gp120 crossreactive antibodies. Journal of Molecular Biology, 369, 696-709. doi:10.1016/j.jmb.2007.01.060

[17] Aires da Silva, F., Corte-Real, S. and Goncalves, J. (2008) Recombinant antibodies as therapeutic agents: Pathways for modeling new biodrugs. BioDrugs, 22, 301-314. doi:10.2165/00063030-200822050-00003 